\title{
Análise Numérica da Ferramenta SIGANATEL para o Cálculo de Viabilidade de Canais de FM
}

\author{
Paulo Henrique da Fonseca Silva e Márcio Galdino Passos
}

\begin{abstract}
Resumo-Neste artigo descreve-se um estudo comparativo para verificar os resultados numéricos da ferramenta SIGANATEL - Sistema de Informações Geográficas da Agência Nacional de Telecomunicações. Neste sentido, a implementação computacional foi realizada em conformidade com as Resoluções da ANATEL, que regulamentam os serviços de radiodifusão de FM. O método proposto possibilita a inclusão de informações a partir do banco de dados digitalizados de relevo da ferramenta SIGANATEL. Os resultados foram obtidos considerando uma estação existente: a rádio Tabajara FM, canal 288 (105,5 MHz), localizada no município de João Pessoa - PB.
\end{abstract}

Palavras-Chave-Radiodifusão, propagação, métodos numéricos.

Abstract-This paper describes an comparative study to verify the numerical results of the SIGANATEL tool - Sistema de Informações Geográficas da Agência Nacional de Telecomunicações. In this purpose, the computational implementation was realized to be in accordance with the ANATEL Resolutions, wich regulate the FM broadcast services. The proposed method makes possible the inclusion of information from the SIGANATEL tool digitalized terrain roughness data banks. The results were obtained considering an existent station: the Tabajara FM radio, 288 channel $(105,5 \mathrm{MHz})$, situated in the João Pessoa city, PB.

Keywords-Broadcasting, propagation, numerical methods.

\section{INTRODUÇÃO}

Ao longo dos anos, os estudos de viabilização de canais de televisão e rádios FM foram realizados com o auxílio de tabelas, curvas, cartas geográficas, calculadoras, etc. Contudo o uso destas ferramentas para a obtenção de dados de um projeto de radiodifusão (perfil do terreno, intensidade de campo do sinal, contornos de serviço, etc.) impõe aos engenheiros projetistas um trabalho minucioso, desde que, uma série de erros podem ser cometidos durante a sua execução. Estes procedimentos manuais são responsáveis pela maior parte do tempo gasto na realização de um projeto.

A ferramenta SIGANATEL é um módulo de cálculo de viabilidade de canais de TV e FM, [1], que reduz em até $70 \%$ o tempo de trabalho dos engenheiros projetistas de sistemas de radiodifusão. Esta ferramenta permite estudos para a viabilização de novos canais e para a alteração dos canais constantes nos Planos Básicos de Distribuição de canais de Televisão em VHF e UHF (PBTV), de Retransmissão de Televisão em VHF e UHF (PBRTV), de Canais de Televisão Digital (PBTVD), de Televisão por Assinatura em UHF

Paulo Henrique da Fonseca Silva e Márcio Galdino Passos, Grupo de Telecomunicações e Eletromagnetismo Aplicado, Centro Federal de Educação Tecnológica da Paraíba, João Pessoa, Brasil, E-mails: henrique@cefetpb.edu.br, marciogpassos@gmail.com.
(PBTVA), e de Radiodifusão Sonora em Freqüência Modulada (PBFM) [2].

Entre as funcionalidades da ferramenta SIGANATEL destaca-se a visualização de mapas com divisões políticas do Brasil e da América Latina, incluindo imagens de satélite e perfis de terreno. Ela acessa o banco de dados da Anatel em tempo real buscando informações técnicas dos canais dos Planos Básicos e realiza o cálculo dos parâmetros necessários para o estudo de viabilidade, tais como: distância ortodrômica, ângulo de azimute, nível médio de radial, contorno protegido e contorno interferente.

A ferramenta SIGANATEL utiliza as curvas de intensidade de campo apresentadas na Recomendação UIT-R P.1546, que apresenta um método de previsões ponto-área para serviços terrestres, na faixa de 30-3000 MHz [3].

Este artigo descreve a aplicação de técnicas numéricas para a solução dos problemas encontrados durante a utilização da ferramenta SIGANATEL para o cálculo de viabilidade de canais. Em particular, foram implementados os seguintes modelos e métodos: i) o modelo da Recomendação UIT-R P.1546-1 utilizado no Brasil desde o ano de 2006, conforme [5], que fornece os valores de intensidade de campo em função da distância a uma emissora e da altura desta acima do nível médio do terreno; ii) o método de Newton [4] usado para a solução do sistema de equações não lineares do modelo que fornece a distância ortodrômica e o ângulo de azimute entre dois pontos localizados por suas coordenadas geográficas na superfície terrestre; iii) o método da falsa posição, usado para a determinação das distâncias aos contornos protegido e interferente de uma estação de radiodifusão.

Um estudo comparativo foi realizado para a verificação dos resultados fornecidos pela ferramenta SIGANATEL. A seção II descreve o regulamento técnico para estações de rádio FM. A seção III descreve o procedimento utilizado em conjunto com as facilidades da ferramenta SIGANATEL. A seção IV apresenta os resultados numéricos obtidos para um estudo de caso considerando a rádio Tabajara FM. As conclusões deste artigo são apresentadas em seguida.

\section{Regulamento TÉCNico PARA EstaÇÕES DE FM}

As implementações computacionais realizadas neste estudo seguem os regulamentos técnicos estabelecidos através das Resoluções da ANATEL, que regulamentam os serviços de radiodifusão de FM no Brasil [5]-[7]. A Recomendação UITR P.1546-1, [3] que serve de base para estas Resoluções da ANATEL, também foi considerada. A metodologia proposta permite a realização de projetos de viabilização de canais de 
forma totalmente digital, com auxílio da ferramenta SIGANATEL e do Matlab 7, programa em que foram simulados os modelos e métodos numéricos estudados.

\section{A. Conceitos Básicos de Radiodifusão de FM}

A cobertura de uma emissora de FM é estabelecida através da intensidade de campo do sinal recebido, definindo-se três áreas de serviço: primária (contorno $74 \mathrm{~dB} \mu$ ), urbana (contorno $66 \mathrm{~dB} \mu$ ) e rural (contorno $54 \mathrm{~dB} \mu$ ). As estações emissoras de FM são enquadradas em classes conforme seus requisitos máximos: de potência efetiva irradiada (ERP), distância máxima ao contorno protegido $(66 \mathrm{~dB} \mu)$, e altura de referência sobre o nível médio do terreno (HNMT).

Uma emissora terá o seu sinal protegido contra interferências prejudiciais dentro de sua área de serviço urbana, delimitada pelo contorno 2 (contorno protegido). A proteção de uma emissora é segurada quando, no seu contorno protegido, a relação entre o sinal desejado e cada um dos sinais interferentes atender as relações de proteção estabelecidas no anexo à Resolução $\mathrm{N}^{\circ}$ 67, 1998 [7]. Existe a possibilidade de interferência quando o contorno interferente de um canal intercepta o contorno protegido de outro canal [5].

\section{B. Levantamento do Nível Médio do Terreno}

No estudo teórico da área de cobertura de uma emissora de FM, suas áreas de serviço são determinadas através de contornos de intensidade de campo do sinal, levando-se em conta um terreno plano de altitude conhecida. As informações de relevo do terreno são levantadas ao longo de trajetos radiais a partir do sistema irradiante. A primeira radial é definida na direção do Norte Verdadeiro (NV) [5].

$\mathrm{O}$ nível médio de uma radial (NMR) corresponde à média aritmética das altitudes do terreno em relação ao nível do mar (cotas), levantadas no trecho entre 3 e 15 quilômetros, em uma radial (quando o contorno de $66 \mathrm{~dB} \mu$ incluir área do território brasileiro) [5]. O nível médio de uma radial deve ser calculado, no mínimo, para 12 radiais com espaçamento angular de $30^{\circ}$ e considerando pelo menos 50 cotas, igualmente espaçadas.

O nível médio do terreno (NMT) é a média aritmética dos níveis médios das radiais. O parâmetro NMT permite que o terreno possa ser considerado plano. Através do NMT calculase a altura h1 = HNMT definida por:

$$
\mathrm{h} 1=\mathrm{HNMT}=\mathrm{HBT}+\mathrm{HT}-\mathrm{NMT}
$$

em que, HBT é a altura da base da torre em relação ao nível do mar; HT é a altura da torre de sustentação até o centro geométrico da antena do sistema irradiante.

\section{Curvas de Intensidade de Campo}

A Resolução da ANATEL $\mathrm{N}^{\circ}$ 398, de 7 de abril de 2005 estabeleceu a adoção exclusiva, a partir do ano de 2006, da Recomendação UIT-R P.1546-1. As tabelas com os valores de intensidade de campo são dadas como uma função da distância no Anexo 4 à Resolução $N^{\circ}$ 398/2005. Dois tipos de curvas de intensidade de campo são considerados para um percurso terrestre: $E(50,50)$ e $E(50,10)$, correspondendo à intensidade de campo excedido em $50 \%$ das localidades e em $50 \%$ e $10 \%$ do tempo, respectivamente.

As curvas $E(50,50)$ são usadas para calcular as distâncias aos contornos de serviço. As curvas $E(50,10)$ são usadas para determinar as distâncias aos contornos interferentes e calcular a intensidade de campo do sinal interferente. Estas curvas de propagação podem ser encontradas no Anexo 3 à Rec. UIT-R P.1546-1 [4].

As curvas de propagação são baseadas em dados medidos e representam os valores de intensidade de campo para uma potência efetiva irradiada (e.r.p.) de $1 \mathrm{~kW}$ nas frequiências nominais de 100, 600 e $2000 \mathrm{MHz}$. A Recomendação UITR P.1546-1 descreve métodos de interpolação/extrapolação dos valores obtidos para os valores nominais de freqüência, HNMT, distância, etc. Além disso, um modelo da intensidade de campo é descrito, possibilitando a implementação computacional do método de previsões ponto-área.

Este modelo foi implementado em Matlab. Os resultados obtidos para $E(50,50)$, percurso terrestre, $\mathrm{f}=100 \mathrm{MHz}$, são apresentados na Figura 1.

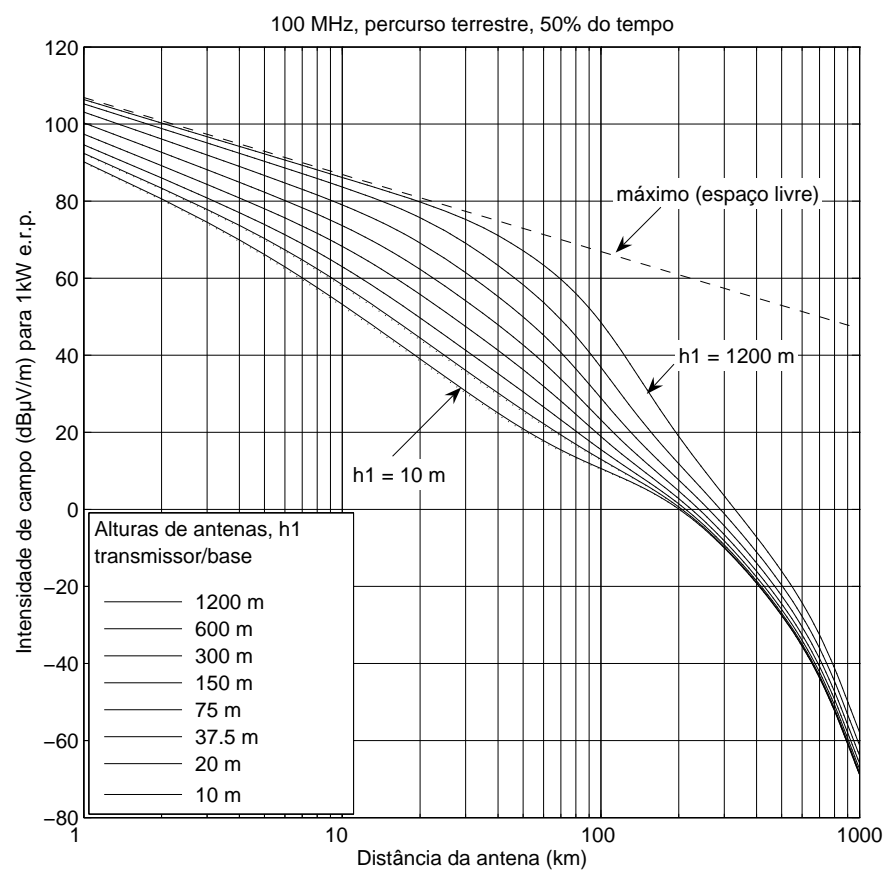

Fig. 1. Curvas $E(50,50)$ para percurso terrestre, $\mathrm{f}=100 \mathrm{MHz}$, em função da distância da antena e da altura h1, e $1 \mathrm{~kW}$ e.r.p.: (- - -) valor máximo, espaço livre; (—) valores calculados, [4]; (...) valores tabelados, [5].

Observa-se na Fig. 1 a concordância entre os valores calculados e os valores tabelados dados em [5], com pequenos erros para valores de h1 entre 10 e $20 \mathrm{~m}$. As curvas indicam os valores de intensidade de campo em $\mathrm{dB}$ acima de $1 \mu \mathrm{V} / \mathrm{m}$ $(\mathrm{dB} \mu)$, para uma e.r.p. de $1 \mathrm{~kW}$, irradiada de um dipolo de meia onda no espaço livre, que produz uma intensidade de campo não atenuada de 221,4 mV/m (aproximadamente $107 \mathrm{~dB} \mu$ ) a $1 \mathrm{~km}$ da antena [3], [5].

\section{Metodologia Utilizada}

A metodologia proposta fundamenta-se na utilização das funcionalidades do SIGANATEL, para a localização ge- 
ográfica de canais existentes no PBFM, cálculo de distâncias, ângulos de azimute, NMT, e cálculo de viabilidade de canais propostos. A fim de se verificar os resultados obtidos com o uso do SIGANATEL, os problemas encontrados são resolvidos através de métodos numéricos.

\section{A. Localização de um Canal Existente}

O primeiro procedimento realizado nesta metodologia foi a localização de um canal existente. Para isto simulou-se uma interferência co-canal entre o canal 288 proposto (canal vago no PBFM) localizado no município de Campina GrandePB e o canal 288 existente (Rádio Tabajara FM) localizado no município de João Pessoa-PB. No menu Cálculos do SIGANATEL obtém-se os canais relevantes ao estudo de viabilidade. Apenas um canal relevante é relacionado, exatamente o canal 288 (105,5 MHz) de João Pessoa.

Os resultados desta aplicação incluem a localização de uma rádio FM e o seu contorno protegido. A distância ao contorno protegido foi obtida criando-se objetos no menu de Ferramentas/Cálculo de Distância/Manual, que representam as radiais 1 e 4, traçada a partir das coordenadas geográficas da Rádio Tabajara FM (07S0825.00, 34W5313.00) na direção do Norte Verdadeiro. O valor da distância ao contorno protegido obtida foi de 29,737 km. A Figura 2 reproduz os resultados fornecidos pelo SIGANATEL.

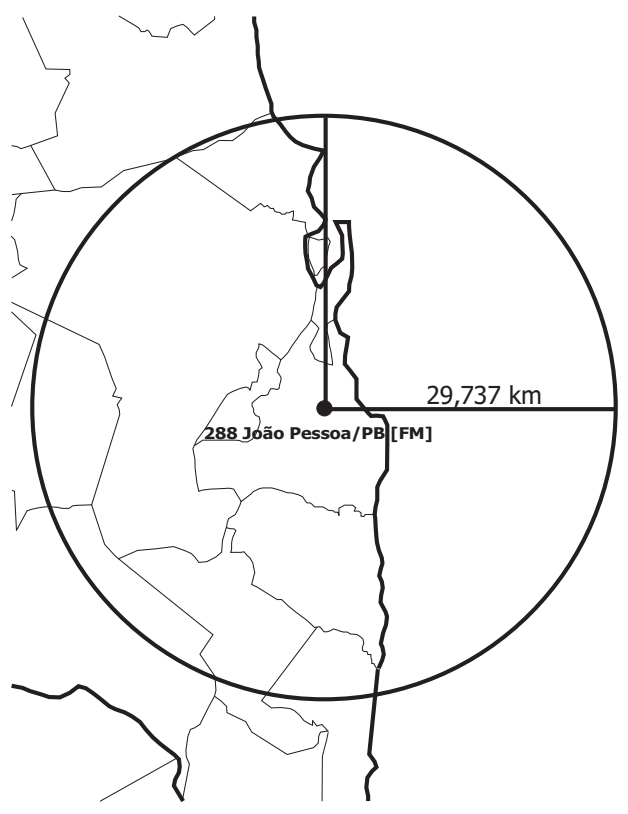

Fig. 2. Localização do canal 288, Rádio Tabajara FM 105,5 MHz.

\section{B. Método Gráfico para o Levantamento de Radiais}

Para usar o banco de dados de relevo da ferramenta SIGANATEL o usuário deve indicar a posição geográfica de cada radial. Os percursos através das radiais são usados para levantar os perfis altimétricos do terreno na área de cobertura, bem como, calcular os níveis médios das radiais.

As radiais $r=2,3,5,6, \ldots, 12$ a partir do local da antena foram definidas através de um método gráfico. Neste caso, as coordenadas geográficas dos pontos extremos de cada radial desejada e das radiais $1\left(0^{\circ}\right)$ e $4\left(90^{\circ}\right)$, mostradas na Fig. 2 , foram relacionadas através de regra de três simples. Por exemplo, os pontos extremos das radiais 2 e 3 são definidos através das expressões:

$$
\begin{aligned}
& l a_{2,3}=l a_{0}+\left(l a_{1}-l a_{0}\right) \cdot \cos (a z) \\
& l o_{2,3}=l o_{0}+\left(l o_{4}-l o_{0}\right) \cdot \operatorname{sen}(a z)
\end{aligned}
$$

em que, $\left(l a_{r}, l o_{r}\right), r=1,2,3,4$ corresponde à coordenada geográfica do ponto de interseção que a radial $r$ faz com a circunferência do contorno protegido; $a z$ é o ângulo de azimute do ponto de origem. Os resultados calculados através de (2) e (3) e usando a simetria do problema serviram para aproximar as radiais com uma distância fixa da antena de 29,737 km. Contudo, as coordenadas geográficas das radiais precisaram ser ajustadas através da interface gráfica do SIGANATEL. A Figura 3 mostra as radiais obtidas.

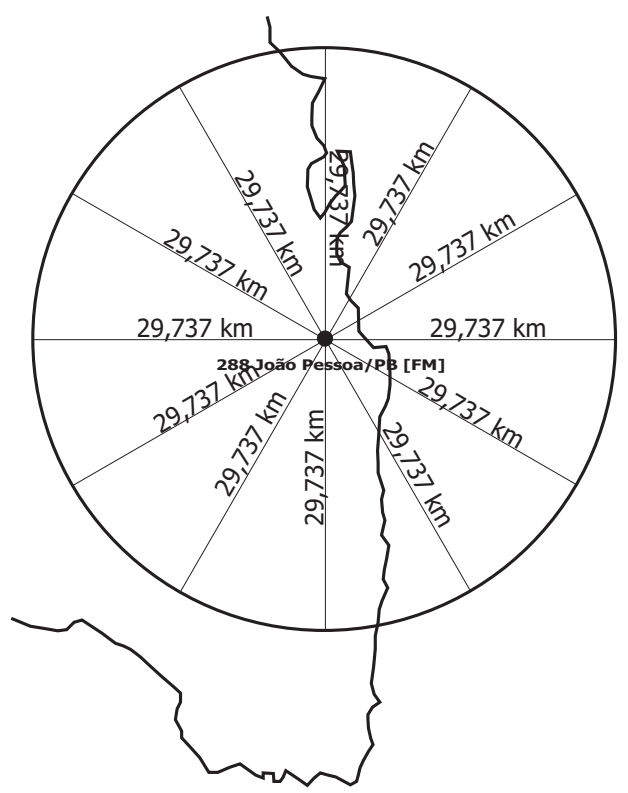

Fig. 3. Localização de 12 radiais, com espaçamento de $30^{\circ}$, no contorno protegido da Rádio Tabajara FM 105,5 MHz.

\section{Método de Newton para o Cálculo de Radiais}

Uma vez que os resultados dos dois métodos usados para levantamento de radiais foram diferentes (usando (2) e (3) e através da interface gráfica do SIGANATEL), um terceiro método mais rigoroso foi implementado para a verificação dos resultados.

Em particular os modelos de distância ortodrômica (4-6), dado em [7], e de ângulo de azimute (7-9) foram solucionadas através do método de Newton.

$$
\begin{gathered}
D=111,1775 \cdot \Delta^{\circ} \\
\Delta^{\circ}=\cos ^{-1}(\Gamma)
\end{gathered}
$$

$\Gamma=\operatorname{sen}\left(l a_{A}\right) \operatorname{sen}\left(l a_{B}\right)+\cos \left(l o_{A}\right) \cos \left(l o_{B}\right) \cos \left(l o_{B}-l o_{A}\right)$ 


$$
\begin{array}{r}
a z_{A}=\pi-\Phi \quad \text { e } \quad a z_{B}=2 \pi-\Phi \\
\Phi=\cos ^{-1}(\Psi) \\
\Psi=\frac{\operatorname{sen}\left(l a_{B}\right)-\operatorname{sen}\left(l a_{A}\right) \cos \left(\Delta^{\circ}\right)}{\operatorname{sen}\left(\Delta^{\circ}\right) \cos \left(l a_{A}\right)}
\end{array}
$$

em que, $\Delta^{\circ}$ é o arco do círculo máximo dado em graus; $\left(l a_{A}, l o_{A}\right)$ e $\left(l a_{B}, l o_{B}\right)$ são as latitudes e longitudes das extremidades de uma dada radial. Conforme a definição de ângulo de azimute, a extremidade $\mathrm{B}$ corresponde ao ponto geográfico situado mais à direita em relação ao meridiano de Greenwich. $\mathrm{O}$ ângulo $\Phi$ formado na extremidade B é medido no sentido anti-horário a partir do NV até a radial. Os ângulos de azimute $\left(a z_{A}, a z_{B}\right)$ são medidos, no sentido horário, a partir do NV até a radial nos pontos $\mathrm{A}$ e $\mathrm{B}$, respectivamente. A Figura 4 ilustra estas definições.

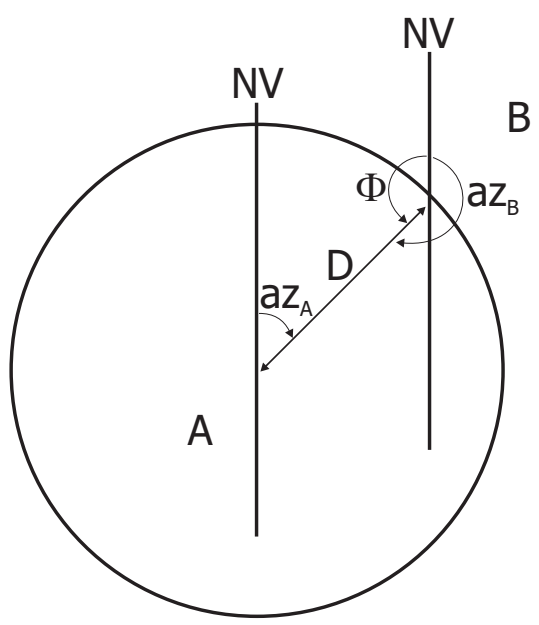

Fig. 4. Distância ortodrômica e ângulos de azimute de uma radial.

Para encontrar as coordenadas geográficas que definem as 12 radiais, o sistema não linear formado por (10-11) foi resolvido numericamente através do método de Newton.

$$
\begin{gathered}
f_{1}\left(l a_{B}, l o_{B}\right)=D r-111,1775 \cdot \cos ^{-1}(\Gamma)=0 \\
f_{2}\left(l a_{B}, l o_{B}\right)=\Phi r-\cos ^{-1}(\Psi)=0
\end{gathered}
$$

em que, $D r$ é a extensão da radial $r$ em km; em $\Phi r$ é o ângulo que estabelece a direção da mesma.

No método iterativo de Newton, uma seqüência convergente de aproximações é encontrada para o sistema não linear (1011) através da seguinte relação recursiva

$$
\mathbf{x}^{(p+1)}=\mathbf{x}^{(p)}-\mathbf{J}^{-1}\left(x^{(p)}\right) \cdot \mathbf{F}\left(x^{(p)}\right)
$$

em que,

$$
\begin{array}{r}
\mathbf{x}=\left[\begin{array}{l}
l a_{B} \\
l o_{B}
\end{array}\right] \text { e } \quad \mathbf{F}=\left[\begin{array}{l}
f_{1} \\
f_{2}
\end{array}\right] \\
\mathbf{J}=\left[\begin{array}{cc}
\frac{\partial f_{1}}{\partial l a_{B}} & \frac{\partial f_{1}}{\partial l o_{B}} \\
\frac{\partial f_{2}}{\partial l a_{B}} & \frac{\partial f_{2}}{\partial l o_{B}}
\end{array}\right]
\end{array}
$$

\section{Determinação da Distância a um Contorno}

O cálculo da distância a um contorno protegido $(C p)$ ou interferente $(\mathrm{Ci})$ envolve o cálculo das raízes das equações não lineares (15-16).

$$
\begin{gathered}
f\left(d_{C p}\right)=C p-E(50,50)=0 \\
f\left(d_{C i}\right)=C i-E(50,10)=0
\end{gathered}
$$

Neste estudo os valores de intensidade de campo foram obtidos através da implementação computacional do modelo descrito em [4]. Através do método da falsa posição as soluções de (15-16) foram encontradas. Este método usa apenas os valores das funções $E(50,50)$ e $E(50,10)$. Neste caso, as soluções iterativas foram encontradas através das relações recursivas (17-18).

$$
\begin{gathered}
d_{C p}(n+1)=d_{C p}(n)-\frac{f\left(d_{C p}(n)\right) \cdot\left(d_{C p}(n)-d_{p}\right)}{f\left(d_{C p}(n)\right)-f\left(d_{p}\right)} \\
d_{C i}(n+1)=d_{C i}(n)-\frac{f\left(d_{C i}(n)\right) \cdot\left(d_{C i}(n)-d_{i}\right)}{f\left(d_{C i}(n)\right)-f\left(d_{i}\right)}
\end{gathered}
$$

em que, $d_{C p}$ e $d_{C i}$ são as distâncias aos contornos protegido e interferente, respectivamente; $d_{p}$ e $d_{i}$ são distâncias fixas; $n=0,1, \ldots$, Nmax; Nmax é o número máximo de iterações.

As distâncias aos contornos $C p$ e $C i$ foram encontradas usando o SIGANATEL em uma simulação de interferência cocanal entre a Rádio Tabajara FM e o canal 288 proposto para o município de Campina Grande-PB. Os resultados gráficos são mostrados na Fig. 5.

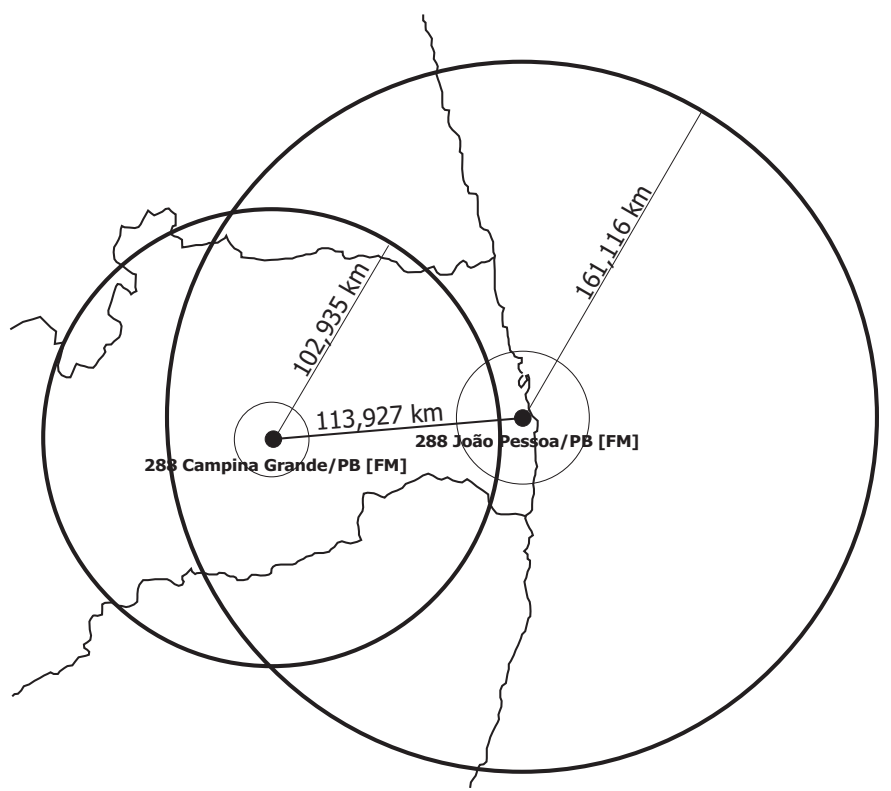

Fig. 5. Distâncias aos contornos teóricos, protegidos e interferentes, para interferência co-canal, simulada com o SIGANATEL entre dois sistemas irradiantes em operação na frequiência 105,5 MHz: i) 288 Campina Grande ERPmáx $=3$ kW, HNMT = 90 m, localização 07S1350.00 / 35W5252.00; ii) 288 João Pessoa - ERPmáx = 15 kW, HNMT = 150 m, localização 07S1350.00 / 35W5252.00.

Por sua vez, usando o modelo de cálculo descrito no Anexo 4 à Rec. UIT-R P.1546-1, [4] em conjunto com o método da 
falsa posição (17-18), as distâncias $d_{C p}$ e $d_{C i}$ aos respectivos contornos foram computadas.

No caso de interferência co-canal a relação de proteção vale $34 \mathrm{~dB}$, [7]. Portanto para um contorno protegido $C p=66$ $\mathrm{dB} \mu$, o contorno interferente vale $C i=32 \mathrm{~dB} \mu$. Os resultados gráficos obtidos para interferência do canal existente no canal proposto são mostrados na Fig. 6. As curvas de intensidade de campo para $f=105,5 \mathrm{MHz}$ foram determinadas através do método de interpolação descrito em [4].

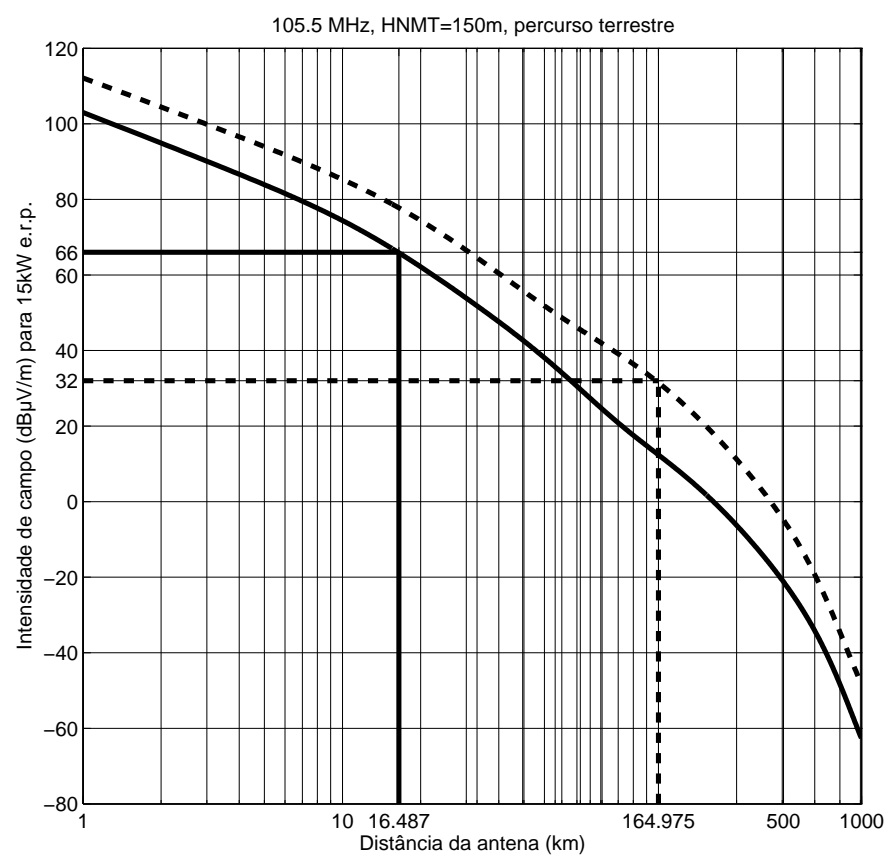

Fig. 6. Determinação das distâncias aos contornos teóricos $C p=66 \mathrm{~dB} \mu$ para o canal proposto e $C i=32 \mathrm{~dB} \mu$ para a Rádio Tabajara FM: (-) $E(50,50)$; (- - ) $E(50,10)$.

\section{RESUltados NumÉricos}

\section{A. Cálculo de Radiais}

A Tabela I apresenta os valores das coordenadas geográficas das radiais obtidas através do método gráfico. Para cada radial, estes resultados são comparados com os valores calculados de distância ortodrômica e de ângulos de azimute através de (4-9). Observa-se a ocorrência de diferenças pequenas entre os resultados do método gráfico e os respectivos valores calculados. Contudo, embora a simulação no SIGANATEL resulte em radiais de mesma extensão $(29,737 \mathrm{~km})$, a aplicação do modelo através de (4-9) aponta para radiais com extensões diferentes. A resposta para esta questão foge ao escopo deste artigo.

A Tabela II apresenta os resultados obtidos através do método de Newton, (10-12). Na aplicação deste método iterativo, o critério de parada $\max \left|F\left(l a_{B}, l o_{B}\right)\right| \leq 10^{-13}$ foi adotado. As coordenadas geográficas obtidas para o traçado das radiais a partir do local da antena foram verificadas através de (4-9), obtendo-se os resultados esperados para $\operatorname{Dr}(\mathrm{km})$ e $a z_{r}\left({ }^{\circ}\right)$.

A Tabela II apresenta os valores encontrados de NMR e de NMT a partir dos dados de relevo gerados pelo SIGANATEL.
Através do menu Ferramentas/Perfil de Terreno do SIGANATEL obtém-se os valores de NMR, bem como, as cotas do perfil do terreno são dadas, no mínimo a cada 90 metros, entre duas localizações geográficas distintas. Para o cálculo dos valores de NMR apresentados na Tabela II, considerou-se 134 cotas para cada trecho de 3 a $15 \mathrm{~km}$ da antena transmissora.

TABELA I

CÁlculo de RAdiais - MÉtodo Gráfico - SigANATEL

\begin{tabular}{|l|c|c|c|c|c|c|}
\hline \multicolumn{5}{|c|}{$\begin{array}{c}\text { Ferramenta } \\
\text { SIGANATEL }\end{array}$} & \multicolumn{2}{c|}{$\begin{array}{c}\text { Valores } \\
\text { Calculados }(4-9)\end{array}$} \\
\hline Radial & Latitude & Longitude & $\operatorname{Dr}(\mathrm{km})$ & $a z_{r}\left({ }^{\circ}\right)$ & Dr $(\mathrm{km})$ & $a z_{r}\left({ }^{\circ}\right)$ \\
\hline 1 & 06S5217,00 & $34 \mathrm{~W} 5113,00$ & 29,7370 & 0,00 & 29,8944 & 0,00 \\
2 & 06S5432,43 & $34 \mathrm{~W} 4258,68$ & 29,7370 & 30,70 & 29,8440 & 29,48 \\
3 & 07S0021,00 & $34 \mathrm{~W} 3713,82$ & 29,7370 & 60,00 & 29,7469 & 60,15 \\
4 & 07S0825,00 & $34 \mathrm{~W} 3503,85$ & 29,7370 & 90,00 & 29,6978 & 89,98 \\
5 & 07S1629,00 & $34 \mathrm{~W} 3713,57$ & 29,7370 & 120,00 & 29,7470 & 119,82 \\
6 & 07S2217,57 & $34 \mathrm{~W} 4258,50$ & 29,7370 & 149,30 & 29,8430 & 150,50 \\
7 & 07S2433,00 & $34 \mathrm{~W} 5113,00$ & 29,7370 & 180,00 & 29,8944 & 180,00 \\
8 & 07S2217,57 & $34 \mathrm{~W} 5927,49$ & 29,7370 & 210,70 & 29,8428 & 209,49 \\
9 & 07S1629,00 & $35 \mathrm{~W} 0512,42$ & 29,7370 & 240,00 & 29,7467 & 240,15 \\
10 & 07S0825,00 & $35 \mathrm{~W} 0722,14$ & 29,7370 & 270,00 & 29,6975 & 269,98 \\
11 & 07S0021,00 & $35 \mathrm{~W} 0512,18$ & 29,7370 & 300,00 & 29,7469 & 299,82 \\
12 & 06S5432,43 & $34 \mathrm{~W} 5927,31$ & 29,7370 & 329,30 & 29,8439 & 330,50 \\
\hline
\end{tabular}

TABELA II

CÁlculo de Radiais - MÉTodo de Newton - NMRs e NMT

\begin{tabular}{|c|c|c|c|c|c|c|}
\hline \multicolumn{5}{|c|}{ Método de Newton (10-12) } & \multicolumn{2}{|c|}{$\mathrm{NMR}(\mathrm{m})$} \\
\hline Radial & Latitude & Longitude & $\operatorname{Dr}(\mathrm{km})$ & $a z_{r}\left({ }^{\circ}\right)$ & SIGANATEL & Calculado \\
\hline 1 & $06 \mathrm{~S} 5222,09$ & $34 \mathrm{~W} 5113,00$ & 29,7372 & 0,00 & 9,00 & 9.3731 \\
\hline 2 & $06 \mathrm{~S} 5431,03$ & $34 \mathrm{~W} 4308,02$ & 29,7372 & 30,00 & 2,00 & 2.9776 \\
\hline 3 & $07 \mathrm{~S} 0023,33$ & $34 \mathrm{~W} 3712,82$ & 29,7373 & 60,00 & 0,00 & 0.0000 \\
\hline 4 & $07 \mathrm{~S} 0824,71$ & $34 \mathrm{~W} 3502,57$ & 29,7370 & 90,00 & 3,00 & 3.1119 \\
\hline 5 & $07 \mathrm{~S} 1626,23$ & $34 \mathrm{~W} 3712,33$ & 29,7370 & 120,00 & 10,00 & 10.4851 \\
\hline 6 & $07 \mathrm{~S} 2218,82$ & $34 \mathrm{~W} 4307,53$ & 29,7369 & 150,00 & 19,00 & 19.2687 \\
\hline 7 & $07 \mathrm{~S} 2427,90$ & $34 \mathrm{~W} 5113,00$ & 29,7369 & 180,00 & 29,00 & 30.0970 \\
\hline 8 & $07 \mathrm{~S} 2218,97$ & $34 \mathrm{~W} 5918,21$ & 29,7369 & 210,00 & 36,00 & 37.1716 \\
\hline 9 & $07 \mathrm{~S} 1626,66$ & $35 \mathrm{~W} 0513,41$ & 29,7368 & 240,00 & 40,00 & 41.0821 \\
\hline 10 & $07 \mathrm{~S} 0825,28$ & $35 \mathrm{~W} 0723,42$ & 29,7367 & 270,00 & 43,00 & 43.5149 \\
\hline 11 & $07 \mathrm{~S} 0023,75$ & $35 \mathrm{~W} 0513,41$ & 29,7370 & 300,00 & 13,00 & 13.4925 \\
\hline 12 & $06 \mathrm{~S} 5431,17$ & 34W5918,21 & 29,7369 & 330,00 & 26,00 & 25.5821 \\
\hline \multicolumn{5}{|c|}{ NMT (m) } & 19,17 & 19,73 \\
\hline
\end{tabular}

\section{B. Viabilidade Técnica do Canal 288, Campina Grande/PB}

A Tabela III contém as informações do canal proposto (canal vago 288, Campina Grande/PB [FM]), bem como, do canal existente (Rádio Tabajara, canal 288, João Pessoa/PB [FM]), conforme os critérios de enquadramento de classe destas estações [7].

\section{TABELA III}

CARACTERÍSTICAS TÉCNICAS DAS ESTAÇÕES

\begin{tabular}{|c|c|c|}
\hline \multicolumn{3}{|c|}{ CANAL PROPOSTO: 288 Campina Grande/PB [FM] } \\
\hline Localização & 07S1350,00 & $35 \mathrm{~W} 5252,00$ \\
\hline CLASSE B1 & ERPmax = 3,00 kW & HNMT =90 m \\
\hline \multicolumn{2}{|c|}{ CANAL EXISTENTE: 288 João Pessoa/PB [FM] } \\
\hline Localização & $07 S 0825,00$ & $34 \mathrm{~W} 5113,00$ \\
\hline CLASSE A3 & ERPmax $=15,00 \mathrm{~kW}$ & HNMT $=150 \mathrm{~m}$ \\
\hline
\end{tabular}

A partir do menu Cálculos/Viabilidade de Canais de FM do SIGANATEL, as distâncias aos contornos protegidos e interferentes foram simuladas. Os resultados são apresentados nas Tabelas IV e V. Eles foram obtidos diretamente através da interface gráfica do SIGANATEL (Método Gráfico), bem como, a partir da opção Relatório do mesmo. Por outro 
lado, os valores calculados foram obtidos através da aplicação do método da falsa posição (17-18), considerando-se uma relação de proteção de $34 \mathrm{~dB}$ para interferência co-canal e um campo interferente de $32 \mathrm{~dB} \mu(0,04 \mathrm{mV} / \mathrm{m})$ [7]. Os contornos encontrados foram ligeiramente diferentes daqueles fornecidos pelo SIGANATEL, levando a uma distância mínima maior entre as estações. Em todos os casos, levando-se em conta a distância existente, os contornos teóricos determinados indicam a inviabilidade do canal proposto.

\section{TABELA IV}

InTERFERÊNCIA Do CANAL EXISTENTE A3 No CANAL Proposto B1

\begin{tabular}{|c|c|c|c|}
\hline \multirow{2}{*}{$\begin{array}{c}\text { Interferência do } \\
\text { Canal Existente A3 } \\
\text { no Canal Proposto B1 }\end{array}$} & \multicolumn{2}{|c|}{ SIGANATEL } & $\begin{array}{c}\text { CALCULADO } \\
(\mathrm{km})\end{array}$ \\
\cline { 2 - 3 } & $\begin{array}{c}\text { Mrátodo } \\
\text { Grico }(\mathrm{km})\end{array}$ & $\begin{array}{c}\text { Relatório } \\
(\mathrm{km})\end{array}$ & \\
\hline Contorno Protegido & 16,737 & 16,387 & 16,487 \\
\hline Contorno Interferente & 161,116 & 161,221 & 164,975 \\
\hline Distância Mínima & 177,853 & 177,608 & 181,462 \\
\hline Distância Existente & 113,927 & 113,927 & 113,781 \\
\hline Viabilidade & Inviável & Inviável & Inviável \\
\hline
\end{tabular}

TABELA V

INTERFERÊNCIA Do CANAL Proposto B1 No CANAL Existente A3

\begin{tabular}{|c|c|c|c|}
\hline $\begin{array}{c}\text { Interferência do } \\
\text { Canal Proposto B1 } \\
\text { no Canal Existente A3 }\end{array}$ & $\begin{array}{c}\text { SIGÁtodo } \\
\text { Gráfico }(\mathrm{km})\end{array}$ & $\begin{array}{c}\text { Relatório } \\
(\mathrm{km})\end{array}$ & $\begin{array}{c}\text { CALCULADO } \\
(\mathrm{km})\end{array}$ \\
\hline Contorno Protegido & 29,737 & 29,772 & 30,002 \\
\hline Contorno Interferente & 102,935 & 103,011 & 105,523 \\
\hline Distância Mínima & 132,672 & 132,783 & 135,525 \\
\hline Distância Existente & 113,927 & 113,927 & 113,781 \\
\hline Viabilidade & Inviável & Inviável & Inviável \\
\hline
\end{tabular}

\section{CONClusões}

Neste artigo, a ferramenta SIGANATEL foi usada para o cálculo de viabilidade de canais de FM. Os resultados obtidos foram analisados em comparação com os modelos e métodos numéricos implementados. No cálculo da distância mínima necessária entre as estações verificou-se uma diferença de $0,1 \%$, entre os resultados gráficos e numéricos fornecidos pela ferramenta SIGANATEL. Em relação aos valores calculados através do modelo da Rec. UIT-R P.1546-1 e dos métodos numéricos propostos neste trabalho, a diferença foi da ordem de $2 \%$. Os contornos de serviço e interferentes calculados usando o modelo da Rec. UIT-R P.1546-1, foram um pouco maiores do que aqueles obtidos usando a ferramenta SIGANATEL. Na computação das NMRs, as diferenças entre os resultados se situaram abaixo de $5 \%$, exceto para a radial 2 , cuja diferença foi de $48,8 \%$. Estas diferenças resultaram num erro porcentual de $2,5 \%$ entre os valores de NMT computados, Tabela II. Os métodos de cálculo de radiais descritos neste artigo podem ser utilizados para possibilitar a inclusão de informações da base de dados de relevo do SIGANATEL, e, portanto, torna possível o cálculo de viabilidade de canais de FM em computadores digitais. Com poucas modificações, os procedimentos descritos neste artigo podem ser aplicados aos planos básicos de distribuição de canais de televisão: PBTV, PBRTV, PBTVD e PBTVA.

\section{REFERÊNCIAS}

[1] SIGANATEL - Sistema de Informações Geográficas (Ferramenta para Cálculo de Viabilidade de Canais de Radiodifusão de TV e FM). Disponível em: : http://sistemas.anatel.gov.br/siganatel/. Acesso em: 20 abr 2007.

[2] ANATEL, "Instruções para Utilização da Ferramenta de Cálculo de Viabilidade de Canais de TV e FM". Disponível em http://www.anatel.gov.br/radiodifusao/instrucoes_siganatel.pdf. Acesso em: 20 abr 2007.

[3] Recomendação UIT-R P.1546-1, "Método de previsões ponto-área para serviços terrestres na faixa de frequiências de 30 a 3000 MHz". Disponível em: www.anatel.gov.br/biblioteca/resolucao/2005/anexoii_res_398_2005.pdf. Acesso em: 15 abr 2007.

[4] Barroso, L. C. et al., "Cálculo Numérico (Com Aplicações)", 2a ed., São Paulo: Harbra, 1987.

[5] Resolução ANATEL ${ }^{\circ}$ 398/2005, 7/4/2005. Disponível em: http://www.anatel.gov.br/biblioteca/. Acesso em: 10 abr 2007.

[6] Resolução ANATEL $n^{\circ}$ 363/2004, 20/4/2004. Disponível em: http://www.anatel.gov.br/biblioteca/. Acesso em: 10 abr 2007.

[7] Resolução ANATEL $n^{\circ}$ 67/1998, 12/11/1998. Disponível em: http://www.anatel.gov.br/biblioteca/. Acesso em: 10 abr 2007. 